\title{
The identification of the cylindrical defect position and size by measuring the radiated sound pressure on the plane
}

Received: 8 June 2017 / Published online: 17 November 2017

(C) The Author(s) 2017. This article is an open access publication

\begin{abstract}
It is important to measure the defect of objects. The radiated sound from the impacted body has many types of information, and it also has the information of the defect. Therefore, in this study, we propose a method in order to identify the position and the size of the defect in the impacted body by measuring the radiated sound pressure. In the proposed method, we calculate the time dependence of the radiated sound pressure from the impacted body with the supposed defect by using FEM analysis. The defect position and size can be given in order to match the time dependence of the sound pressure with the measured sound pressure. In order to obtain much information involved in the defect, it is necessary to measure the sound pressure in the wide range. However, it is difficult to prepare hundreds of microphones to measure the radiated sound distribution. Thus, we constructed the measuring system of the sound pressure distribution by moving a microphone and applying the same impact force to the body. In this system, we can measure fictionally the sound pressure distribution on the plane over $200 \mathrm{~mm} \times 200 \mathrm{~mm}$ area with $2 \mu \mathrm{m}$ resolution. The efficiency of the proposed method is confirmed by some experiments.
\end{abstract}

\section{Introduction}

Inverse problems to determine the impact force have been studied. Inoue et al. identified the impact force by the strain response caused in the elastic body subjected to an impact force [1-3]. The impact force of the composite materials are identified by a piezoelectric sensor [4-13]. On the other hand, the radiated sound from the impacted body must have the information with respect to the impact force. Tsuji et al. proposed methods to identify the impact force by using the measured sound pressure and the relationship between the impact force and the radiated sound [14-17]. The method to identify the impact position by using the impact sound is studied in [17-19]. On the other hand, the determination of the defect is important, and the radiated sound from the impacted body must have the information of the defect in the impacted body.

In this study, we propose a method to identify the size and the position of the defect in the impacted body by measuring the radiated sound pressure from the impacted body. In the proposed method, the time dependence of the radiated sound pressure from the impacted body, which has the supposed defect and is subjected to the known impact force, is calculated by using FEM analysis. The defect position and size can be given in order to match the time dependence of the sound pressure with the measured sound pressure. Much information, such as a hundred of microphone arrays, should be required, since the determination of the defect should be the confusing problem. However, such a microphone array system has not been constructed. Thus, we construct

S. Iino $\cdot$ S. Yahiro $\cdot$ T. Nishikawa $\cdot$ T. Tsuji $(\bowtie)$

Department of Precision Mechanics, Chuo University, Kasuga 1-13-27, Bunkyo-ku, Tokyo 112-8551, Japan

E-mail: tsuji@mech.chuo-u.ac.jp

S. Iino

E-mail: a12.r4s3@g.chuo-u.ac.jp 


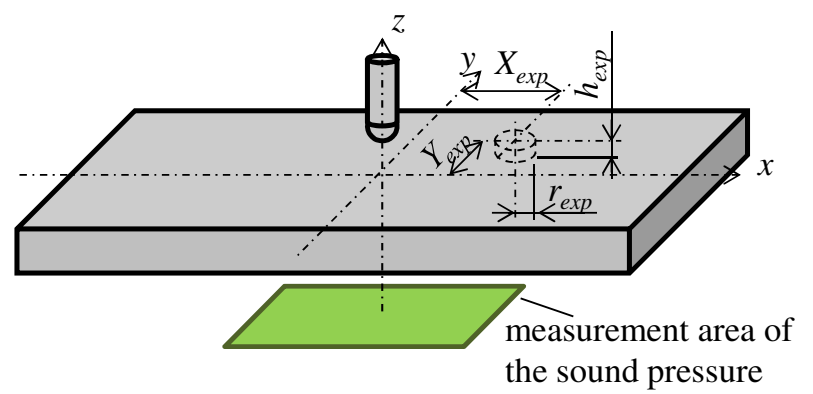

Fig. 1 The impacted body of the plate with the cylindrical defect

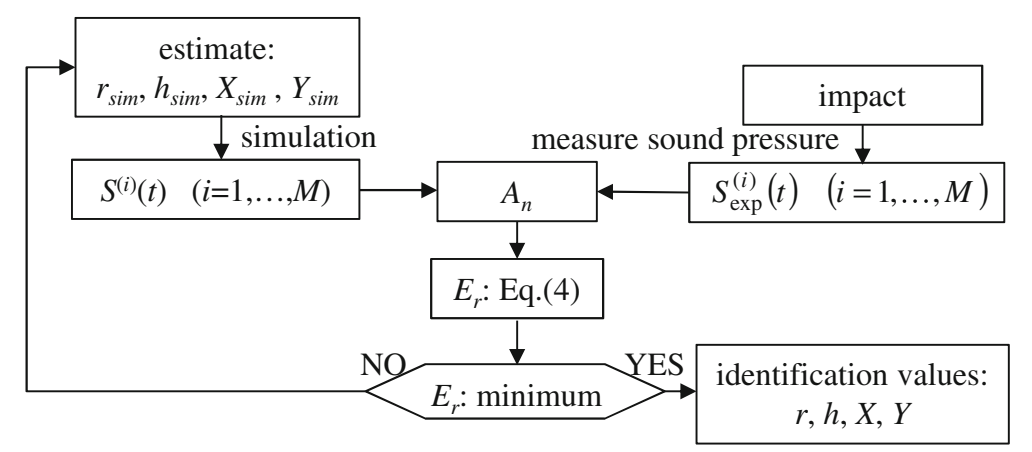

Fig. 2 The flowchart to identify the defect position and size

the measuring system to measure the radiated sound pressure distribution by moving a microphone. By this system, the sound pressure is measured on the plane over $200 \mathrm{~mm} \times 200 \mathrm{~mm}$ area with $2 \mu \mathrm{m}$ resolution. By using these sound data and FEM analysis, the size and the position of a defect can be identified.

\section{Identification principle of the defect position and size}

We consider the problem that the impact force is applied to the plate with the cylindrical defect as shown in Fig. 1. The cylindrical defect position and size are identified by comparing the measured sound pressure to the simulated sound pressure. The center of the top surface of the plate is set as the origin of the coordinates. The actual defect radius, depth, and position are defined as $r_{\exp }, h_{\exp }$ and $\left(X_{\exp }, Y_{\exp }\right)$, respectively, and the supposed defect radius, depth, and position are defined as $r_{\operatorname{sim}}, h_{\operatorname{sim}}$ and $\left(X_{\operatorname{sim}}, Y_{\text {sim }}\right)$, respectively. The time dependence of the known impact pressure is defined as follows:

$$
p_{\text {sim }}(t)=p_{0}\left[\frac{1}{2}\left\{1-\cos \left(\pi \frac{t}{T}\right)\right\} H(t) H(T-t)+H(t-T)\right]
$$

where $T, p_{0}$ and $H(t)$ are the rise time, the magnitude of the impact pressure, and the Heaviside step function, respectively. The arbitrary impact pressure $p(t)$ can be given by $p_{\text {sim }}(t)$ and unknown coefficient $A_{n}$ as follows:

$$
p(t)=\sum_{n=1}^{N} A_{n} p_{\operatorname{sim}}\left(t-t_{n}\right) \quad\left(t_{n}=\Delta t(n-1) \frac{K}{N}\right)
$$

where $\Delta t, K$, and $N$ are the time step, the number of the measurement points, and the number of convolution, respectively.

The time dependence of the sound pressure $S_{\mathrm{sim}}^{(i)}(t)(i=1,2, \ldots, M)$ at point $i=1-M$ can be given by the FEM analysis when the impact pressure $p_{\text {sim }}(t)$ is applied to the plate with the cylindrical defect. Since the impact pressure and the impact sound pressure have a linear relationship, the time dependence of the sound pressure $S^{(i)}(t)$ by $p(t)$ can be given by using $S_{\text {sim }}^{(i)}(t)$, as follows:

$$
S^{(i)}(t)=\sum_{n=1}^{N} A_{n} S_{\mathrm{sim}}^{(i)}\left(t-t_{n}\right) \quad(i=1,2, \ldots, M)
$$



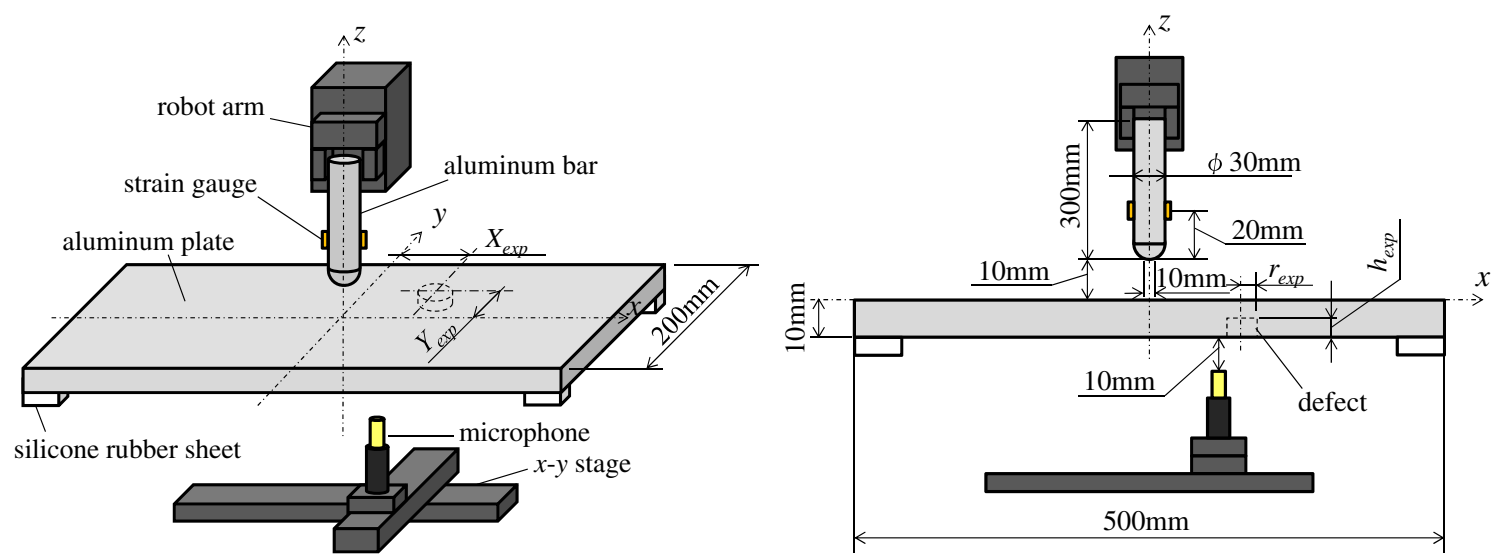

(a)

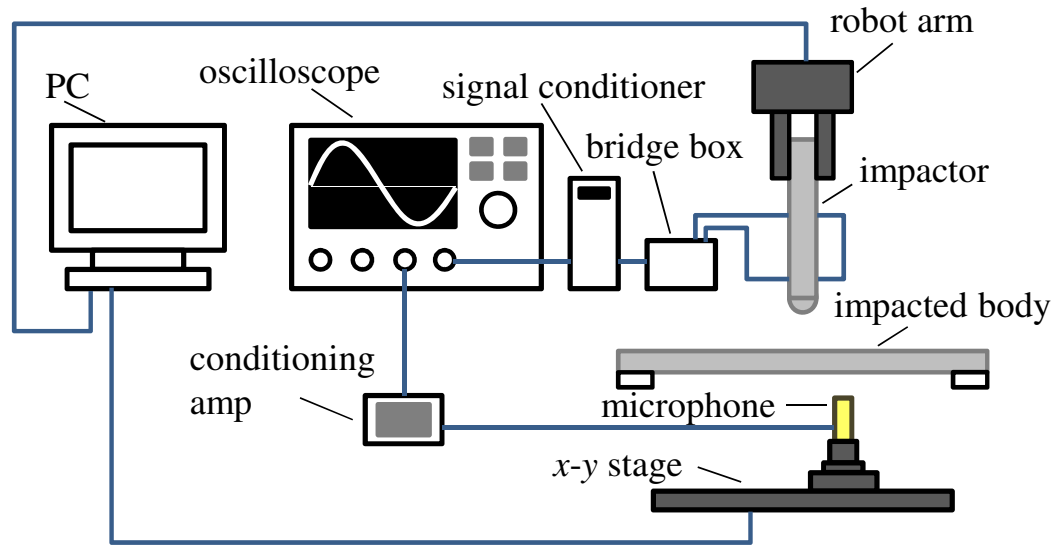

(b)

Fig. 3 The measuring system of the plane sound pressure distribution from the impacted body. a The impactor, impacted body, and the microphone moving system. b The outline of the measuring system

The mean square of the difference between the calculated sound pressure $S^{(i)}(t)$ and the measured sound pressure $S_{\exp }^{(i)}(t)$ over the measured points can be given as follows:

$$
E_{r}=\frac{1}{M} \sum_{i=1}^{M} \sum_{k=1}^{K}\left\{S^{(i)}\left(t_{k}\right)-S_{\exp }^{(i)}\left(t_{k}\right)\right\}^{2}=\frac{1}{M} \sum_{i=1}^{M} \sum_{k=1}^{K}\left\{A_{n} S_{\text {sim }}^{(i)}\left(t_{k}-t_{n}\right)-S_{\exp }^{(i)}\left(t_{k}\right)\right\}^{2}
$$

where $t_{k}(k=1,2, K)$ and $K$ are the measurement time and the total number of the measurement time of the sound pressure, respectively. According to the least squares method, $A_{n}$ can be given as the solution of simultaneous linear equations as follows:

$$
\sum_{n=1}^{N}\left\{\sum_{i=1}^{M} \sum_{k=1}^{K} S_{\text {sim }}^{(i)}\left(t_{k}-t_{n}\right) S_{\text {sim }}^{(i)}\left(t_{k}-t_{m}\right)\right\} A_{n}=\sum_{i=1}^{M} \sum_{k=1}^{K} S_{\exp }^{(i)}\left(t_{k}\right) S_{\text {sim }}^{(i)}\left(t_{k}-t_{m}\right) \quad(m=1,2, \ldots, N) .
$$

Figure 2 shows the flowchart through the measurement of the impact sound pressure to the identification of the defect position and size. We can obtain $r_{\text {sim }}, h_{\text {sim }}$ and $\left(X_{\text {sim }}, Y_{\text {sim }}\right)$ which minimize $E_{r}$ and consider these values as the identified defect size $r, h$, and position $(X, Y)$.

\section{Impact sound pressure distribution measuring system}

In order to determine the confusing system such as the defect, as much information as the plane distribution of the radiated sound pressure should be obtained. However, the microphone system which can measure the sound 


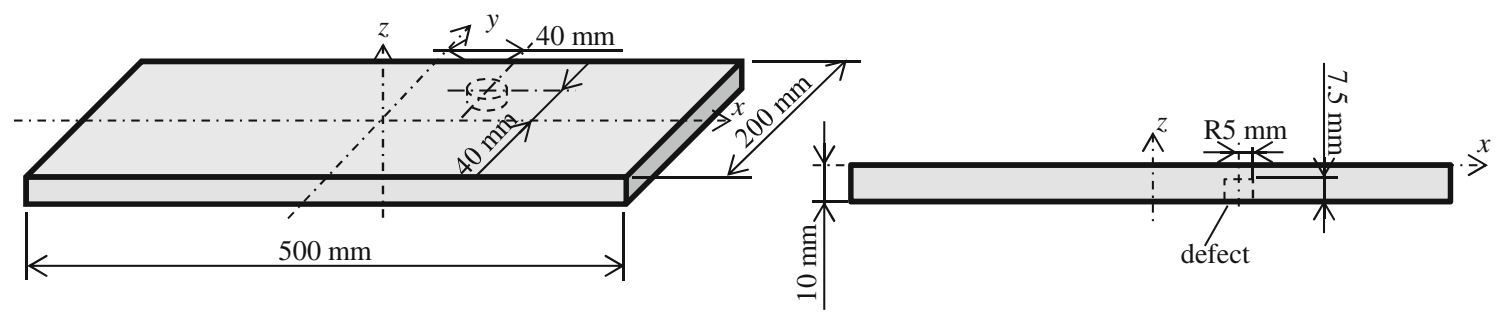

Fig. 4 The actual defect parameters $\left(r_{\exp }=5 \mathrm{~mm}, h_{\exp }=7.5 \mathrm{~mm}, X_{\exp }=40 \mathrm{~mm}\right.$ and $\left.Y_{\exp }=40 \mathrm{~mm}\right)$

pressure distribution on the plane has not been developed yet. Thus, instead of the plane microphone system, we constructed the measuring system of the radiated sound pressure distribution by moving the microphone as shown in Fig. 3a. The sound distribution with $2 \mu \mathrm{m}$ resolution over $200 \times 200 \mathrm{~mm}$ area can be measured by the present microphone system. The aluminum bar $(\phi 30 \times 300 \mathrm{~mm})$ is used as the impactor. The tip side of the aluminum bar is rounded to be circular plane with the diameter $10 \mathrm{~mm}$, and the aluminum plate (500 $\times 200 \times 10 \mathrm{~mm}$ ) supported by the silicone rubber sheets with thickness $5 \mathrm{~mm}$ is used as the impacted body. By lifting the bar to a certain height $(10 \mathrm{~mm})$ and releasing by the robot arm, the same impact force is applied to the center of the plate automatically. A microphone (Bruel and Kjaer, Type 4190) is installed on the $x-y$ stage, which moves with every impact interval as a step. Thus, the radiated sound pressure distribution under the below surface of the plate can be measured. The robot arm and the $x-y$ stage are controlled by a PC as shown in Fig. 3b. The sound pressure from the microphone is recorded by the oscilloscope.

In order to confirm the reproducibility of the equipment, we perform the experiment in which the impactor is dropped repeatedly to the center of the plate, and the sound pressures are measured. The number of iterations is 1000. The standard deviation with respect to the maximum values of the measured sound pressures is $102.3 \pm 1.9 \mathrm{~Pa}$ in the $95 \%$ CI. Namely, the scatter was $2 \%$ or less. By this system, we can measure the sound pressure distribution with sufficient accuracy.

We perform the impact experiment by using the plate as shown in Fig. $4\left(r_{\exp }=5 \mathrm{~mm}, h_{\exp }=\right.$ $7.5 \mathrm{~mm}, X_{\exp }=40 \mathrm{~mm}$ and $Y_{\exp }=40 \mathrm{~mm}$ ). Figure 5 shows the sound pressure distribution on a plane $z=-20 \mathrm{~mm}, x=-50$ to $50 \mathrm{~mm}$ and $y=-50$ to $50 \mathrm{~mm}$ with $10 \mathrm{~mm}$ resolution at the time $t=0.050$ $0.185 \mathrm{~ms}$. The impact pressure is observed under the impacted point $(0,0)(\mathrm{mm})$ as shown in Fig. 5a and spreads concentrically as shown in Figs. 5b-d. When the sound pressure reaches the peak, the center becomes a concave distribution as shown in Fig. 5e-i, because the plate returns to the original state. Finally, as shown in Fig. 5i, the sound pressure in the neighborhood at $(40,40 \mathrm{~mm})$ takes a maximum value. This placement is coincident with the defect position.

\section{Identification of the position and the size of the defect}

The position and the size of the defect are identified by the proposed method in Sect. 2. We carry out the dynamic FEM analysis coupled with the plate deformation and the sound pressure by using the finite element method analysis software ANSYS (ANSYS Inc.). The relationship between the known impact pressure $p_{\text {sim }}(t)$ in Eq. 1 as shown in Fig. 6 and the sound pressure $S^{(i)}(t)$ at microphone $i$ is given by the FEM analysis. Table 1 shows the mechanical properties of the aluminum plate and the air. Figure 7 shows the FEM analysis model. The model is made of the aluminum plate $(500 \times 200 \times 10 \mathrm{~mm})$ with the cylindrical defect and the air $(500 \times 300 \times 100 \mathrm{~mm})$ surrounding the plate. An FEM analysis is carried out repeatedly with changing the defect size and position in order to identify the defect.

It is a complicated problem to determine the position and the size of the defect. Thus, only the position of the defect is identified at first. The size of the cylindrical defect is known with $r_{\text {sim }}=r_{\exp }=5 \mathrm{~mm}$, $h_{\text {sim }}=h_{\exp }=7.5 \mathrm{~mm}$. The unknown parameter is the position of the defect $\left(X_{\text {sim }}, Y_{\text {sim }}\right)$. The value of $E_{r}$ in Eq. (4) is calculated with respect to $X_{\text {sim }}$ and $Y_{\text {sim }}$ with $\Delta t=0.005 \mathrm{~ms}, K=50$, and $N=20$. The sound pressure was measured on the plane $x=-50$ to $50 \mathrm{~mm}, y=-50$ to $50 \mathrm{~mm}$ as shown in Fig. 8 . In order to define the efficiency of the measurement points, various measurement points are used as shown in Fig. 8a-d.

Figure 9 shows the distribution of $E_{r}$ with respect to $\left(X_{\text {sim }}, Y_{\text {sim }}\right)$ by the number of the measurement points $M=4$ to 121 as shown in Fig. 8. The value of $E_{r}$ does not take any minimum point in Fig. 9a, since the number of measurement points is only four points. In Fig. 9b, the minimum point can be seen, but the value of $E_{r}$ 


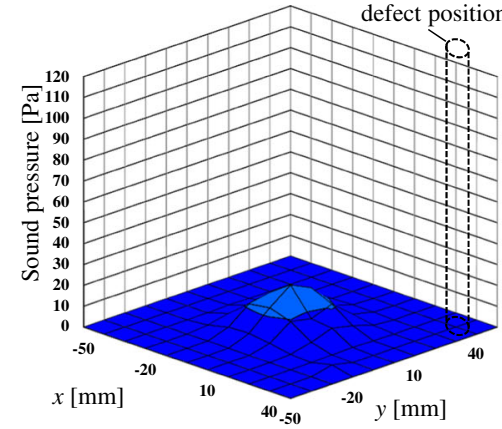

(a)

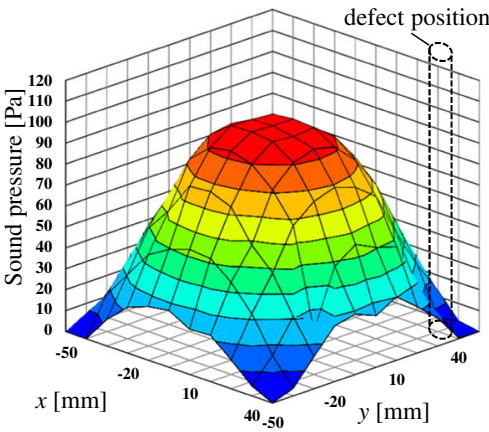

(d)

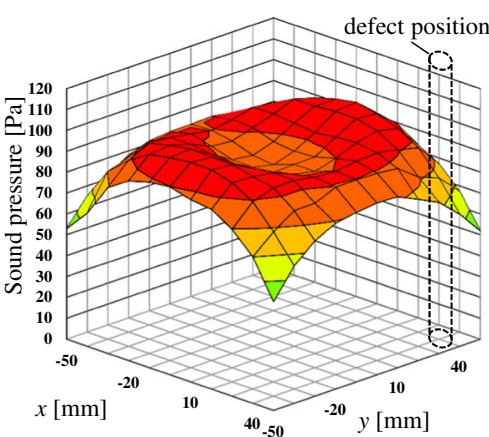

(g)

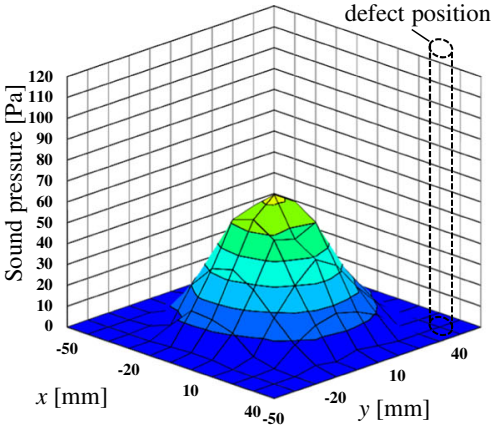

(b)

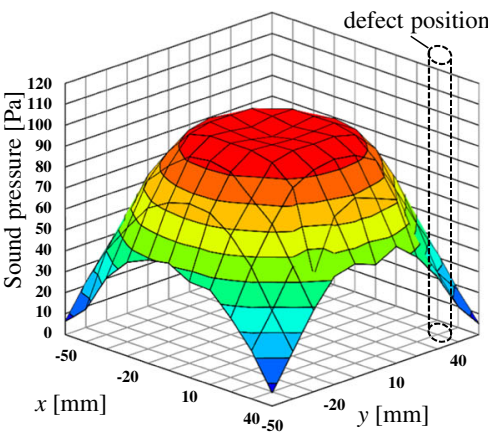

(e)

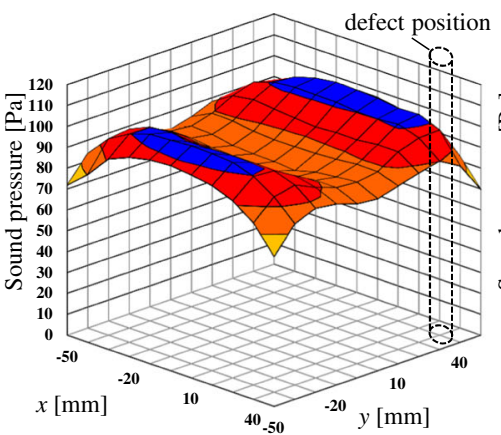

(h)

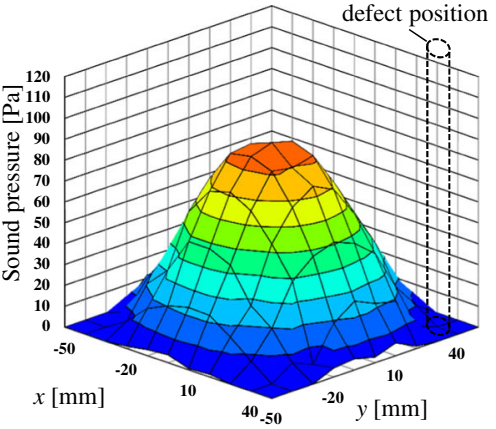

(c)

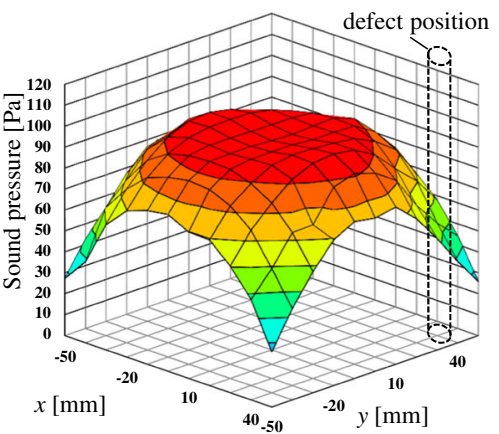

(f)

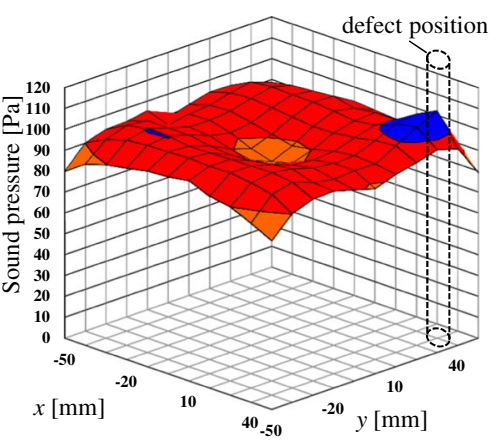

(i)

Fig. 5 The distribution of the sound pressure at time $t=0.05-0.185 \mathrm{~ms}$. ( $z=-20 \mathrm{~mm}, x=-50$ to $50 \mathrm{~mm}, y=-50$ to 50 $\mathrm{mm})$. a Time $t=0.065(\mathrm{~ms})$. b Time $t=0.080(\mathrm{~ms})$. c Time $t=0.095(\mathrm{~ms})$. d Time $t=0.110(\mathrm{~ms})$. e Time $t=0.125(\mathrm{~ms})$. f Time $t=0.140(\mathrm{~ms})$. g Time $t=0.155(\mathrm{~ms})$. h Time $t=0.170(\mathrm{~ms})$. i Time $t=0.185(\mathrm{~ms})$

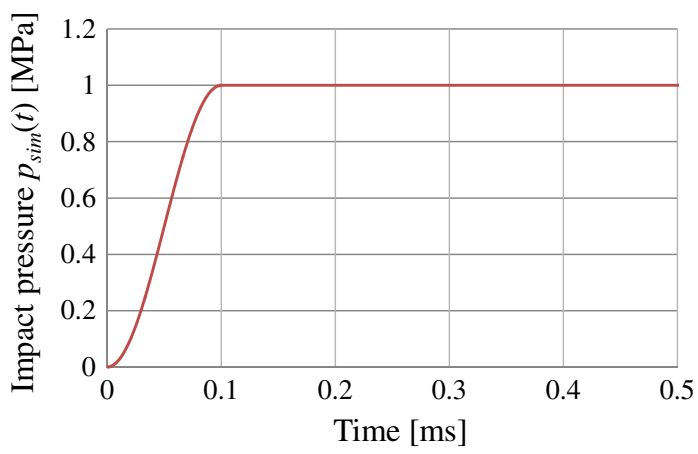

Fig. 6 The time dependence of the pressure as the input for the FEM analysis 
Table 1 Mechanical properties of the aluminum plate and the air for the FEM analysis

\begin{tabular}{llc}
\hline Material & Aluminum & Air \\
\hline Density & $2700 \mathrm{~kg} / \mathrm{m}^{3}$ & $1.3 \mathrm{~kg} / \mathrm{m}^{3}$ \\
Poisson's ratio & 0.33 & - \\
Young's modulus & $68 \mathrm{GPa}$ & - \\
Acoustic velocity & - & $346 \mathrm{~m} / \mathrm{s}$ \\
\hline
\end{tabular}

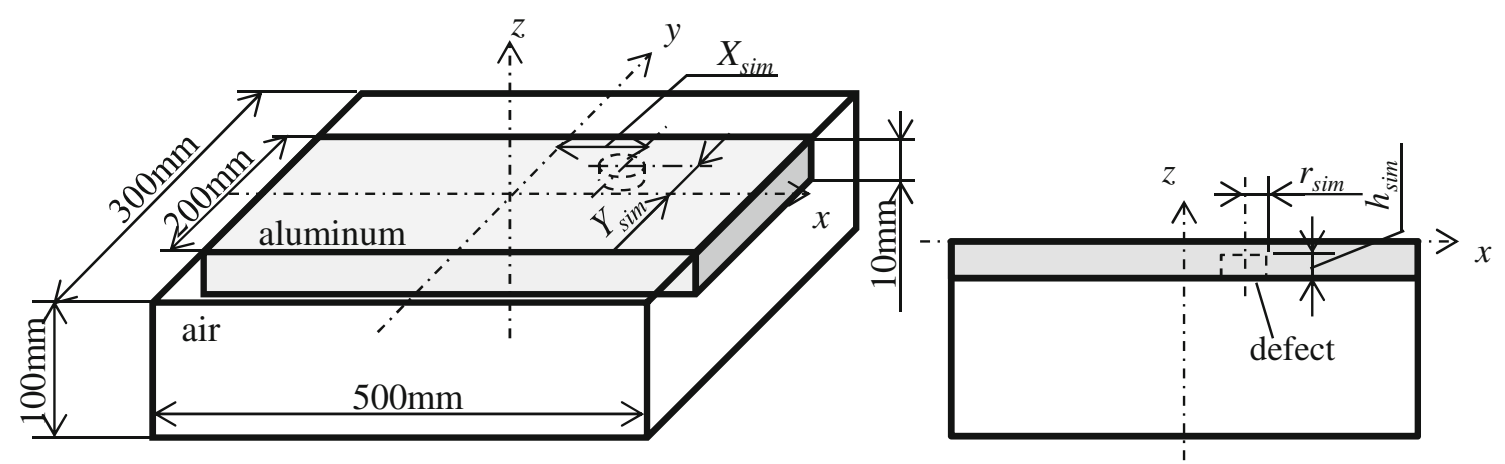

Fig. 7 FEM analysis model of the plate with the air
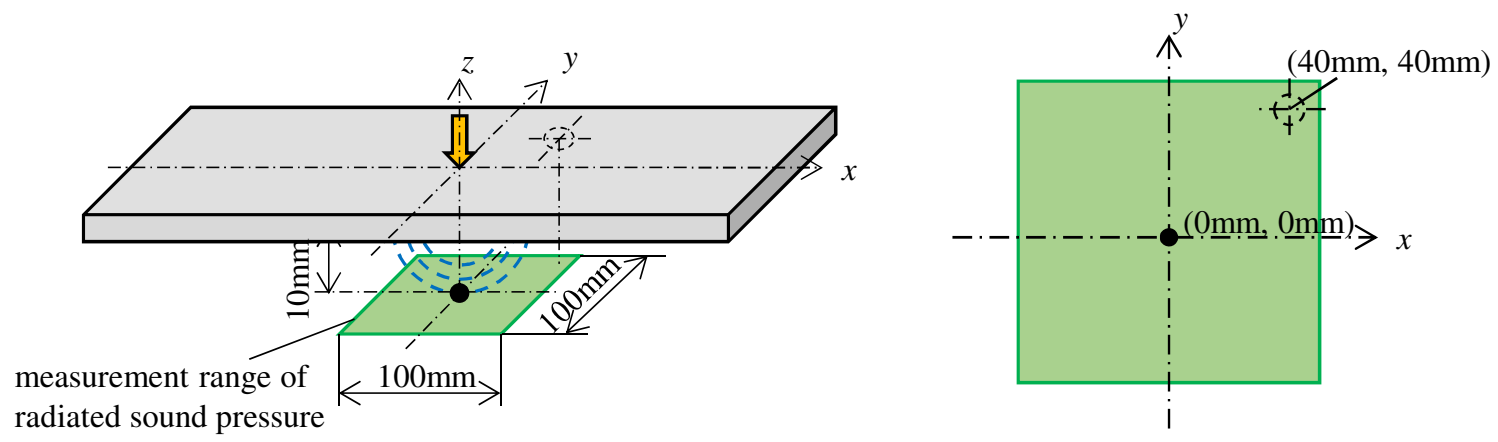

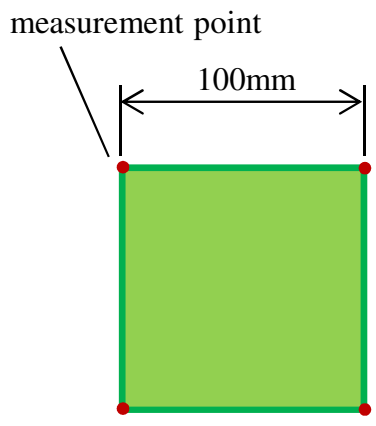

(a)

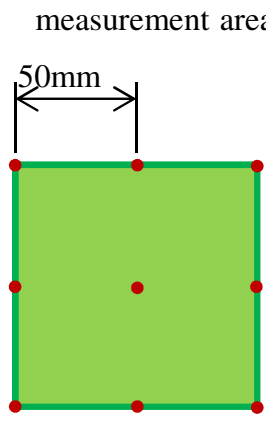

(b)

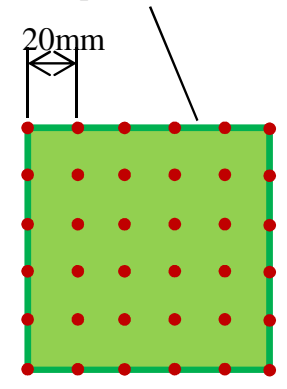

(c)

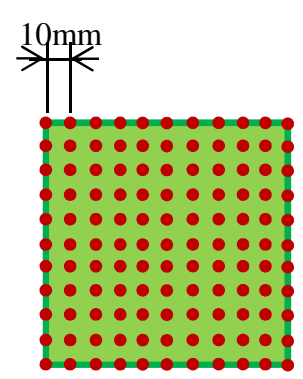

(d)

Fig. 8 The measurement range and points of the radiated sound pressure $(x= \pm 50 \mathrm{~mm}, y= \pm 50 \mathrm{~mm}, 10 \mathrm{~mm}$ below the plate, actual defect position $\left.X_{\exp }=40 \mathrm{~mm}, Y_{\exp }=40 \mathrm{~mm}\right)$. a $M=4 . \mathbf{b} M=9$. $\mathbf{c}=36 . \mathbf{d}=121$

takes almost same value, and it is difficult to determine the minimum point clearly. In Fig. 9c, d, the minimum point can be determined clearly. Thus, more than $20 \mathrm{~mm}$ measurement resolution of the sound pressure should be required to determine the defect position by the present method. The identified position of the cylindrical defect for the various sound pressure resolutions is listed in Table 2. The large number of the measurement points is required to obtain an accurate identification. 

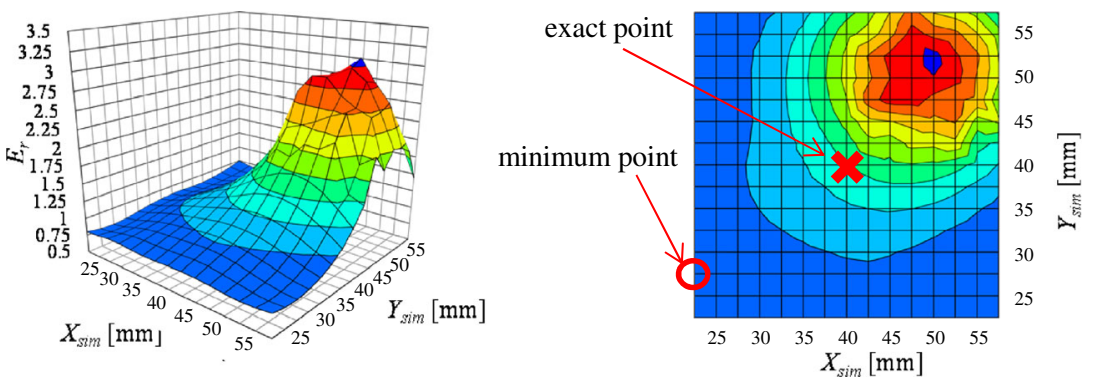

(a)
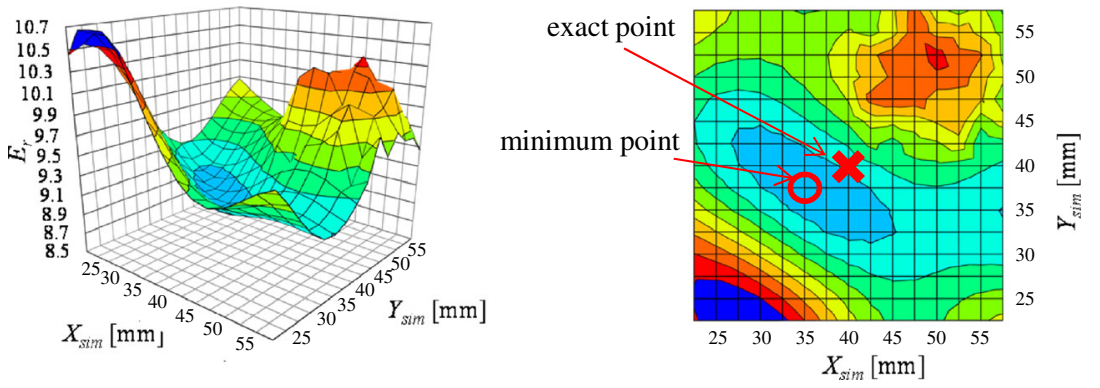

(b)
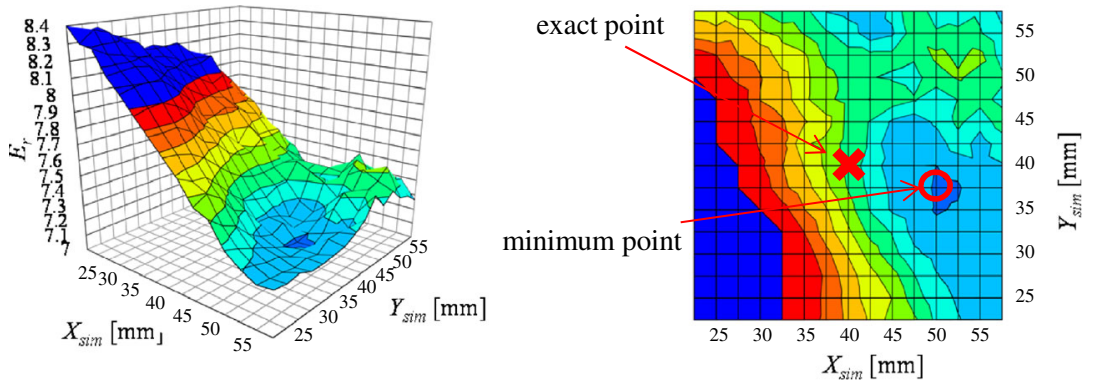

(c)
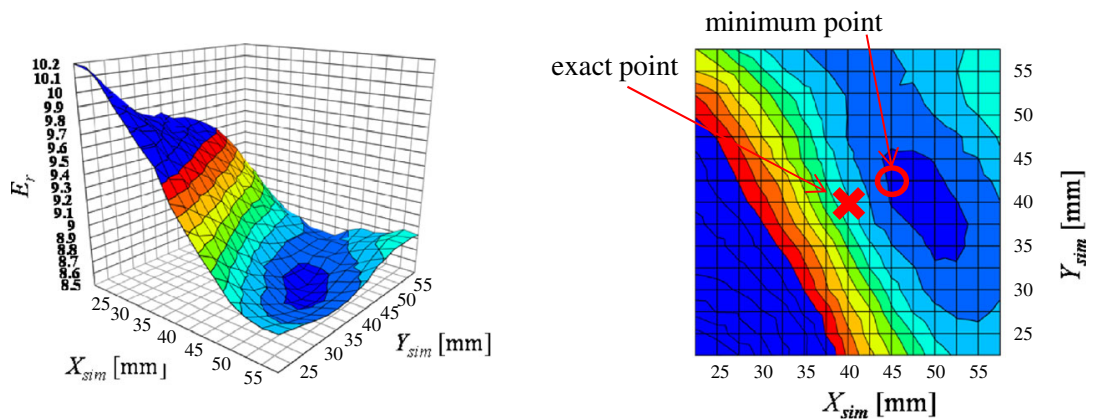

(d)

Fig. 9 The distribution of $E_{r}$ with respect to $X_{\text {sim }}, Y_{\text {sim }}\left(r_{\operatorname{sim}}=r_{\exp }=5 \mathrm{~mm}, h_{\text {sim }}=h_{\exp }=7.5 \mathrm{~mm}\right.$ ) (sound pressure measurement area: $y= \pm 50 \mathrm{~mm}, x= \pm 50 \mathrm{~mm}, 10 \mathrm{~mm}$ below the plate). a Measurement resolution of sound pressure distribution: $100 \mathrm{~mm}(M=4)$. b Measurement resolution of sound pressure distribution: $50 \mathrm{~mm}(M=9)$. c Measurement resolution of sound pressure distribution: $20 \mathrm{~mm}(M=36)$. d Measurement resolution of sound pressure distribution: $10 \mathrm{~mm}(M$ $=121$ )

The position $x$ and size of the defect are identified by using the plate with $r_{\exp }=10 \mathrm{~mm}, h_{\exp }=5 \mathrm{~mm}$ and $\left(X_{\exp }, Y_{\exp }\right)=(70,0)(\mathrm{mm})$. The position along the $y$ direction of the cylindrical defect is known with $Y_{\text {sim }}=Y_{\text {exp }}=0 \mathrm{~mm}$. The unknown parameters are the size of the defect $r_{\text {sim }}, h_{\text {sim }}$, and the position $X_{\text {sim }}$. The minimum values of these unknown parameters is determined by calculating $E_{r}$ by changing the values of $r_{\text {sim }}, h_{\text {sim }}$, and $X_{\text {sim }}$ with the resolutions of 1,1 , and $5 \mathrm{~mm}$, respectively. In order to consider the effect of the 
Table 2 The identified defect position for the measurement points $M=4-121$. The size of the defect is known as $r_{\mathrm{sim}}=r_{\mathrm{exp}}=5$ $\mathrm{mm}, h_{\mathrm{sim}}=h_{\exp }=7.5 \mathrm{~mm}$. The actual defect position is $X_{\exp }=40 \mathrm{~mm}$ and $Y_{\exp }=40 \mathrm{~mm}$. The sound pressure measurement area is $x=-50$ to $50 \mathrm{~mm}, y=-50$ to $50 \mathrm{~mm}$

\begin{tabular}{|c|c|c|c|c|}
\hline$\sharp$ & Resolution (mm) & $M$ & $X(\mathrm{~mm})$ & $Y(\mathrm{~mm})$ \\
\hline 1 & 100 & 4 & - & - \\
\hline 2 & 50 & 9 & 35 & 37.5 \\
\hline 3 & 20 & 36 & 50 & 37.5 \\
\hline 4 & 10 & 121 & 45 & 42.5 \\
\hline
\end{tabular}

Table 3 The identified defect size and position $\left(Y_{\text {sim }}=Y_{\exp }=0 \mathrm{~mm}\right.$, measurement area: $y=-50$ to $\left.50 \mathrm{~mm}\right)$

\begin{tabular}{|c|c|c|c|c|c|c|}
\hline$\#$ & Measurement area $(\mathrm{mm})$ & Resolution (mm) & $M$ & $r(\mathrm{~mm})$ & $h(\mathrm{~mm})$ & $X(\mathrm{~mm})$ \\
\hline 1 & -50 to 50 & 10 & 121 & 4 & 1 & 45 \\
\hline 2 & -50 to 50 & 5 & 441 & 5 & 1 & 45 \\
\hline 3 & -10 to 90 & 10 & 121 & 9 & 1 & 65 \\
\hline 4 & -10 to 90 & 5 & 441 & 9 & 3 & 70 \\
\hline 5 & -90 to 90 & 10 & 209 & 9 & 1 & 70 \\
\hline 6 & -90 to 90 & 5 & 777 & 9 & 7 & 70 \\
\hline
\end{tabular}

measurement area and resolution of the sound pressure, these known parameters are obtained for the various values of the measurement area and resolutions of the sound pressure and are listed in Table 3. The identified position $X$ is not in good agreement with the actual value $X=70 \mathrm{~mm}$ for $\sharp 1,2,3$ in Table 3 , because the measurement area is not including the defect position. The identified value of $h$ is not in good agreement with the actual value $h=5 \mathrm{~mm}$ for $\sharp 5$, since the sound measurement resolution is not satisfied. Consequently, for the good identification of the defect, it is required to measure the sound pressure with wide area and precise resolution.

\section{Conclusions}

We proposed a method to determine the position and the size of the cylindrical defect in the impacted plate by using the sound pressure distribution from the impacted body. In order to confirm the present method, the equipment, by which the sound pressure distribution can be measured fictionally, is constructed. By this equipment, the defect position and size can be determined using the proposed method. For the good identification of the defect, it is required to measure the sound pressure with wide area and precise resolution.

Open Access This article is distributed under the terms of the Creative Commons Attribution 4.0 International License (http:// creativecommons.org/licenses/by/4.0/), which permits unrestricted use, distribution, and reproduction in any medium, provided you give appropriate credit to the original author(s) and the source, provide a link to the Creative Commons license, and indicate if changes were made.

\section{References}

1. Inoue, H., Ishida, H., Kishimoto, K., Shibuya, T.: Measurement of impact load by using an inverse analysis technique (comparison of methods for estimating the transfer function and its application to the instrumented Charpy impact test). Trans. Jpn. Soc. Mech. Eng. 57, 424-429 (1991)

2. Inoue, H., Kishimoto, K., Shibuya, T., Koizumi, T.: Estimation of impact load by inverse analysis: optimal transfer function for inverse analysis. Trans. Jpn. Soc. Mech. Eng. 57, 2727-2734 (1991)

3. Inoue, H., Ikeda, N., Kishimoto, K., Shibuya, T., Koizumi, T.: Inverse analysis of the magnitude and direction of impact force. Trans. Jpn. Soc. Mech. Eng. 59, 572-579 (1993)

4. Atobe, S., Hu, N., Fukunaga, H.: Real-time identification of impact force on plates using experimental transfer matrices. Jpn. Soc. Aeronaut. Space Sci. 67, 48-55 (2012)

5. Tajima, M., Matsumoto, S., Fukunaga, H.: Impact force identification of CFRP laminated plates using PZT piezoelectric sensors (2nd report, experimental verification). Trans. Jpn. Soc. Mech. Eng. 70, 1747-1754 (2004) 
6. Tracy, M., Chang, F.-K.: Identifying impacts in composite plates with piezoelectric strain sensors. J. Intell. Mater. Syst. Struct. 9, 929-937 (1998)

7. Seydel, R., Chang, F.-K.: Impact identification of stiffened composite panels: I. System development. Smart Mater. Struct. 10, 354-369 (2001)

8. Hu, N., Matsumoto, S., Nishi, R., Fukunaga, H.: Identification of impact forces on composite structures using an inverse approach. Struct. Eng. Mech. 27, 409-424 (2007)

9. Wang, B., Takatsubo, J., Akimune, Y., Tsuda, H.: Development of a remote impact damage identification system. Struct. Control Health Monit. 12, 301-314 (2005)

10. Fukunaga, H., Umino, T., Hu, N.: Impact force identification of CFRP stiffened panel under multiple loading. Struct. Health Monit. 1, 177-184 (2007)

11. Park, J., Chang, F.-K.: Monitoring impact events using a system-identification method. AIAA J. 47, 2011-2021 (2009)

12. Atobe, S., Fukunaga, H., Hu, N.: Impact force identification of CFRP structures using experimental transfer matrices. Comput. Mater. Contin. 26, 67-90 (2011)

13. Gaul, L., Hurlebaus, S.: Determination of the impact force on a plate by piezoelectric film sensors. Arch. Appl. Mech. 69, 691-701 (1999)

14. Tsuji, T., Kawada, Y., Suzuki, Y., Yamaguchi, T., Noda, N.: Identification of an impact force by radiated sound from the impacted body (non-contact measuring experiments of the identification by the inverse analysis). Jpn. Soc. Aeronaut. Space Sci. 65, 701-707 (1999)

15. Tsuji, T., Kurimoto, T., Shibuya, T.: Determination of the impact force at a plate by using acoustic response and FEM analysis. Trans. Jpn. Soc. Mech. Eng. 74, 858-863 (2008)

16. Tsuji, T., Kurimoto, T., Shibuya, T.: An identification method of the time dependence of the impact force by using acoustic response and FEM analysis. In: Irschik, H., Krommer, M., Watanabe, K., Furukawa, T. (eds.) Mechanics and Model-Based Control of Smart Materials and Structures. Springer, Vienna. https://doi.org/10.1007/978-3-211-99484-9_19

17. Tsuji, T., Itou, Y., Shimada, H., Tominaga, Y.: An identification of the impact position, the impact force, the Young modulus of the impacted body and the radius of the impact by using acoustic response and FEM analysis. Trans. Jpn. Soc. Mech. Eng. 77, 1433-1443 (2011)

18. Canistraro, H.A., Jordan, E.H.: Projectile-impact-location determination: an acoustic method. Meas. Sci. Technol. 7, 17551760 (1996)

19. Atobe, S., Kobayashi, H., Hu, N., Fukunaga, H.: Real-time impact force identification of CFRP laminated plates using sound waves. In: Proceedings of the 18th International Conference on Composite Materials (2011) 